\title{
Syndecan-1 expression in ovarian endometrioid cancer tissue has negative correlation with estrogen status
}

\author{
Alexander V. Volchek \\ Novosibirsk State Medical University \\ Novosbirsk, Russia \\ alexander@volcheck.ru
}

\author{
Dmitriy V. Morozov \\ City Clinical Hospital №1 \\ Novosibirsk, Russia \\ mdvil07@mail.ru
}

\author{
Yulia S. Timofeeva \\ Novosibirsk State Medical University \\ Novosbirsk, Russia \\ dr.yustimofeeva@gmail.com \\ Igor O. Marinkin \\ Novosibirsk State Medical University \\ Novosbirsk, Russia \\ rectorngmu@yandex.ru
}

\author{
Yanina M. Evseeva \\ Novosibirsk State Medical University \\ Novosbirsk, Russia \\ janinaevseeva@yahoo.com \\ Svetlana V. Aidagulova \\ Novosibirsk State Medical University \\ Novosibirsk, Russia \\ s.aydagulova@gmail.com
}

\begin{abstract}
Cancer treatment shifts from mono-targeted to combination therapy, considering the tumor microenvironment with heparan sulfates, including Syndecan-1 (SDC1). The aim of this work was to study correlation between core-protein SDC1 and estrogen receptors expression in surgical material of $\mathbf{1 5}$ women aged 53,0 $\pm 10,9$ years with endometrioid ovarian cancer (EOC) using immunohistochemistry and automatic image analysis. The staining intensity of SDC1 in tumor tissue was $1304,23 \pm 65,85$ units, the staining intensity of the estrogen receptors as $1137,88 \pm 135,40$ units. The significant inverse correlation $(R=-\mathbf{0 , 5 1 2}, p=0,003)$ between SDC1 and estrogen receptors expression levels were obtained. Most likely, this deals with progressive character of EOC.
\end{abstract}

Keywords - ovarian endometrioid cancer, syndecan-1, estrogen receptors, tumor tissue, immunohistochemistry

\section{Motivation and Aim}

Ovarian cancer has the 3 rd place among all gynecological neoplasms but is one of the leading causes of death in the structure of gynecological oncology worldwide [1]. Status of estrogen receptors was shown to play crucial role for the progression of OEC and being predictor of the response to hormone therapy [2]. The lack of expression of these receptors is associated with low-grade tumors, which are more aggressive and have poor prognosis. Nowadays, it is expected that as cancer treatment shifts from mono-targeted to combination therapy, the management of biosynthesis and degradation of heparan sulfates (of which SDC1 is a representative) are likely to become the main new direction of cancer therapy [3]. The aim of this work was to study correlation between SDC1 and estrogen receptors expression in women with EOC.

\section{Methods and Algorithms}

A clinical and morphological study of 15 women with EOC aged 40 to 77 years (mean age 53,0 $\pm 10,9$ years) was carried out. The diagnosis of EOC was based on specific endometrioid histotype of surgical samples with positive expression of immunohistochemical markers: Cytokeratin 7, PAX 8, Vimentin and estrogen and progesterone receptors. Women with pregnancy and severe immunodeficiency were excluded. In all cases the informed consent of the patient to the examination and treatment of EOC was taken in accordance with the directives of the European Community (86/609 / EEC) and the Helsinki Declaration, in compliance with the «Ethical Principles for Scientific Medical Research with Human Participation» and in accordance with the «Rules of Clinical Practice in Russian Federation». Surgical tumor samples were fixed in $10 \%$ buffered formalin solution. For immunohistochemistry, $5-\mathrm{mkm}$ serial sections of formalinfixed, paraffin-embedded tissue sections were deparaffinized and antigens were retrieved. The mouse monoclonal antiSDC1 and rabbit monoclonal anti-Estrogen Receptor primary antibodies (ThermoScientific, cat. № MS-1793RQ and RM91-50, Clone SP1, respectively) were used. For negative control, the primary antibodies were replaced by $5 \%$ bovine serum. Immunostaining patterns were visualized using UltraVision Quanto Detection System HRP (ThermoFisher Scientific). The sections were counterstained with Hematoxylin and observed by light microscopy using Axio Scope.A1 microscope with the camera AxioCam MRc5 and software ZEN blue. For the quantitative analysis the average intensity of the intra- and extracellular DAB-positive products was estimated, taking into account the area of the staining zone in pixels and the relative gray gradations by the recommended formula [4] minimum of 50 images per antibody with magnification $40 \times 10$ (Zeiss, Oberkochen, Germany). Statistical processing of the results was performed using the statistical software application package of StatSoft inc. (2011). STATISTICA (data analysis software system) version 10.www.statsoft.com. A value of $p<0,05$ was considered to indicate a statistically significant difference. Due to small amount of patients nonparametric statistic methods were used. Correlations were evaluated using Spearman rank R. Comparing of intensity values (staining concentrations) of SDC1 and estrogen receptors were done with Wilcoxon test.

\section{Results}

The optimal cytoreductive surgery was performed for 10 patients, in the volume of complete cytoreduction in 3 cases and in suboptimal cytoreduction in 2 cases. After surgical staging according to the FIGO classification, 2nd stage of EOC was diagnosed in 4 patients, 3rd stage in 10 patients and 1 woman had stage 4 . Microscopically tumor tissue in the surgical material consisted of a large number of fused glands or cribriform proliferations of spindle-shaped cells, oval or tubular pseudoglands, which had a clear luminal edge, lined with stratified, mucin-free epithelium. Some areas have destructive growth with obvious stromal invasion consists of cells or group of cells which infiltrated randomly the desmoplastic stroma. Secretory changes were resemble the 
early phase of endometrial secretion; sometimes in the stroma luteinized cells were detected. In EOC samples the automated image analysis revealed the SDC1 core-protein staining intensity as $1304,23 \pm 65,85$ units (varying from 1281,02 to 1335,71). The estrogen receptors in tumor tissue were expressed in average as $1137,88 \pm 135,40$ units (varying from $1024,73$ to 1239,84 units $)(p=0,003)$. The significant inverse correlation between SDC1 and estrogen receptors expression levels were obtained $(\mathrm{R}=-0,512)$. Cancer progression is closely related to interactions between cancer cells and tumor microenvironment that are in a dynamic interplay and are regulated by extracellular matrix. Overexpression of SDC1 has been reported in breast, pancreatic, prostate, ovarian, and endometrial cancer [5].

\section{Conclusion}

The significant inverse correlation $(\mathrm{R}=-0,512$, $\mathrm{p}=0,003)$ between SDC1 and estrogen receptors expression levels were obtained. Most likely, this concerns the progressive feature of the EOC, which requires further researches.

\section{ACKNOWLEDGMENT}

The study was carried out as part of the State Assignment of the Ministry of Health of Russian Federation for research and development № 056-00141-18-00 (2018 - 2020).

\section{REFERENCES}

[1] Torre L.A. et al. (2018) Ovarian cancer statistics. CA Cancer J. Clin. 68: 284-296.

[2] Piperigkou Z., Karamanos N.K. (2019) Estrogen receptor-mediated targeting of the extracellular matrix network in cancer. Semin Cancer Biol. pii: S1044-579X(19)30088-4.

[3] Nagarajan A. et al. (2018) Heparan sulfate and heparan sulfate proteoglycans in cancer initiation and progression. Front Endocrinol (Lausanne). 9: 483.

[4] Bouzin C. et al. (2016) Digital pathology: elementary, rapid and reliable automated image analysis. Histopathology. 68(6):888-896.

[5] Palaiologou M. et al. (2014) CD138 (syndecan-1) expression in health and disease. Histology and Histopathology. 29(2): 177-189. 